\title{
Baicalein induces cell apoptosis via the MAPK pathway in anaplastic thyroid cancer cells
}

Eun Sook Kim, Se Eun Han, Il Seong Nam-Goong and Young Il Kim

Department of Internal Medicine, Ulsan University Hospital, College of Medicine University of Ulsan

\section{ABSTRACT}

Objective: In this present study, we aimed to investigate the potential effects of baicalein on FRO anaplastic thyroid cancer cells and the underlying mechanisms through which baicalein exerts its action.

- Methods: Cell viability was measured in FRO cells by MTT assay. The expression of apoptotic proteins, Bax, cleaved caspase-3, PARP, Cox-2, and MAP kinases, ERK, JNK, and p38 in FRO cells by Western blot, respectively.

- Results: : . Cell viability assay was indicated that baicalein potently suppressed the cell growth in a time- and dose-dependent manner. We also found that baicalein can induce the expression of apoptotic proteins, Bax, cleaved caspase-3, PARP, and Cox-2 and the phosphorylation of ERK, JNK, and p38 MAPK in a dose-dependent manner. Moreover, baicalein decreased the expression levels of E-cadherin and N-cadherin in FRO cells..

- Conclusion: These findings suggested that baicalein can induce apoptosis in anaplastic thyroid cancer cells through inhibition of the MAPK pathway

\section{INTRODUCTION}

- Anaplastic thyroid cancer (undifferentiated, less than $5 \%$ of all cases of thyroid cancers) is one of the most aggressive forms of malignancies which grow very rapidly. Tumors are poorly defined. Although several conventional medications have been applied for the treatment of anaplastic thyroid cancer, but current therapies still rather limited and novel therapeutic strategies are require
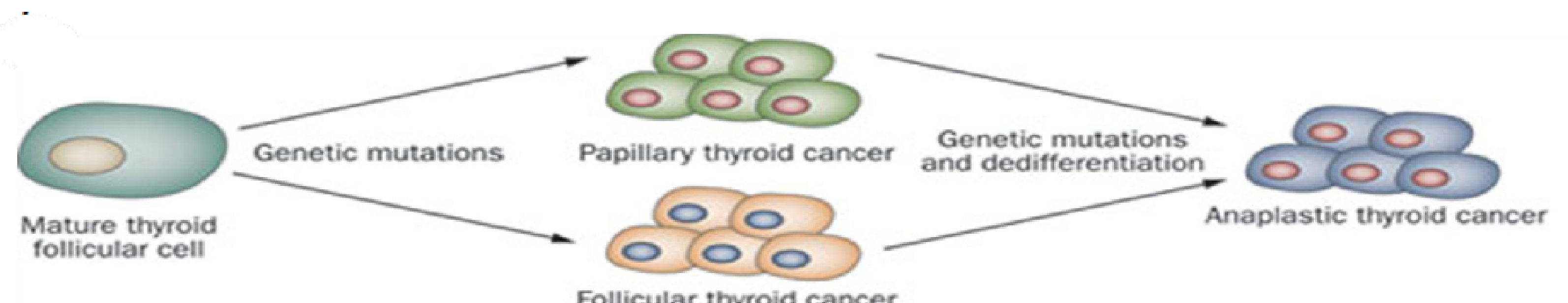

- Baicalein is a flavone, a type of flavonoid, originally isolated from the roots of Scutellaria baicaiensis and S. lateriflora, and is known various biological properties such as antiinflammation, antioxidation, antiallergy, anticancer, and neuroprotection.

- In cancer study, the inhibitory effects on growth and invasion/metastasis in colorectal, gastric, hepatocellular, pancreatic cancers were reported.

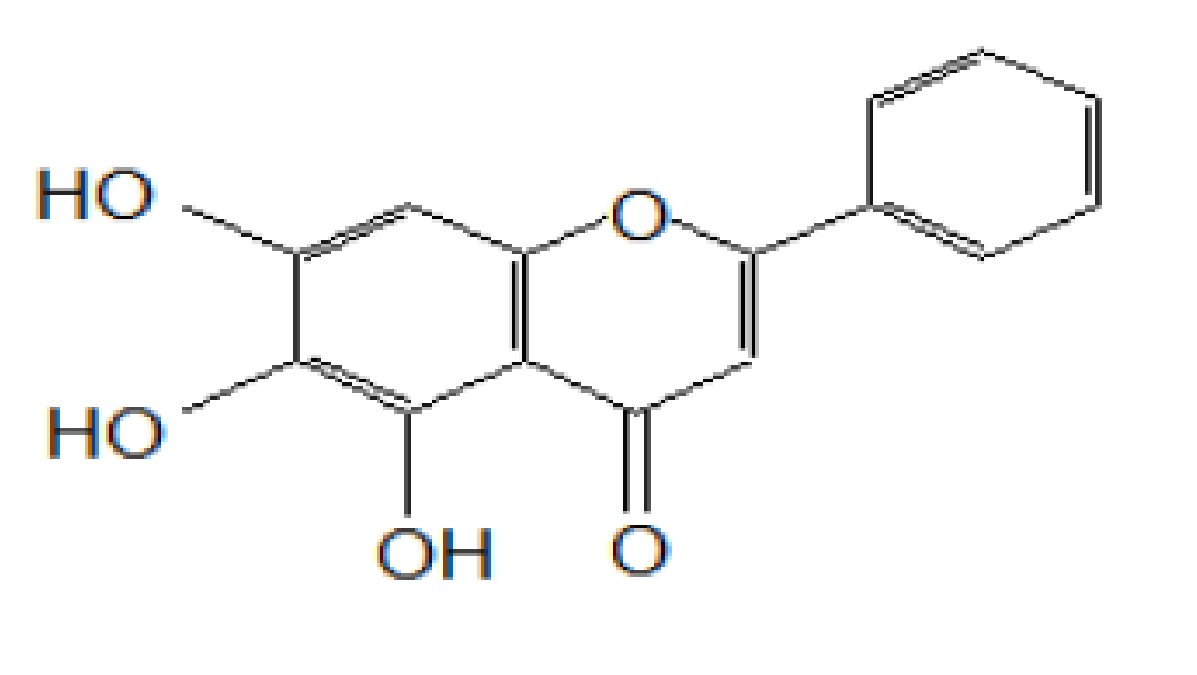

BAICALEIN

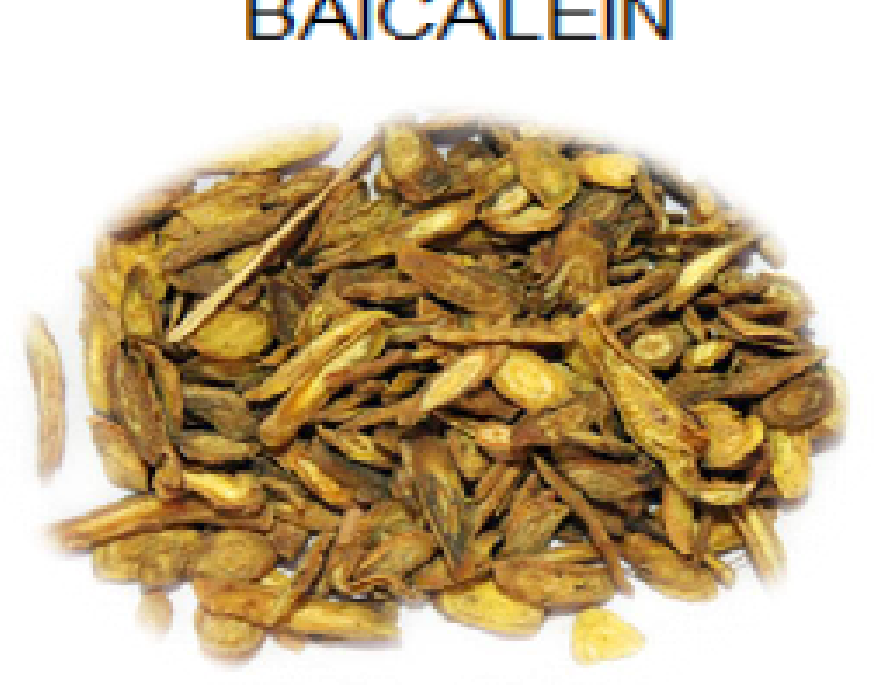

\section{RESULTS}

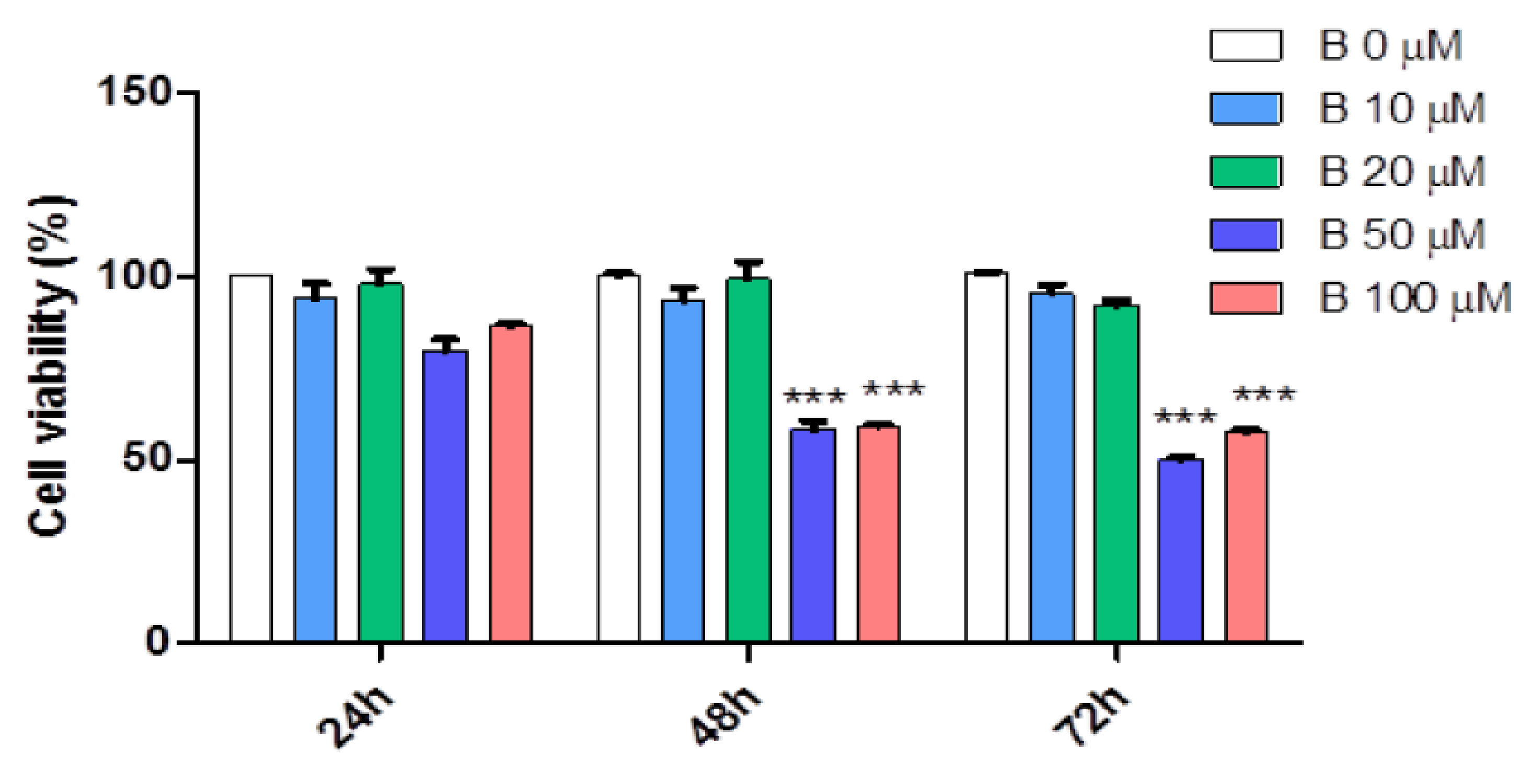

Figure 1. Effect of baicalein on cell viability in FRO cells Cells were treated with baicalein (B) for $24 \mathrm{~h}, 48 \mathrm{~h}$, and $72 \mathrm{~h}$. Cell viability was measured by MTT assay. Values are the means \pm SEMs of three independent experiments. ${ }^{* * *} \mathrm{P}<0.001$ vs. normal cells $(\mathrm{B} 0 \mu \mathrm{M})$

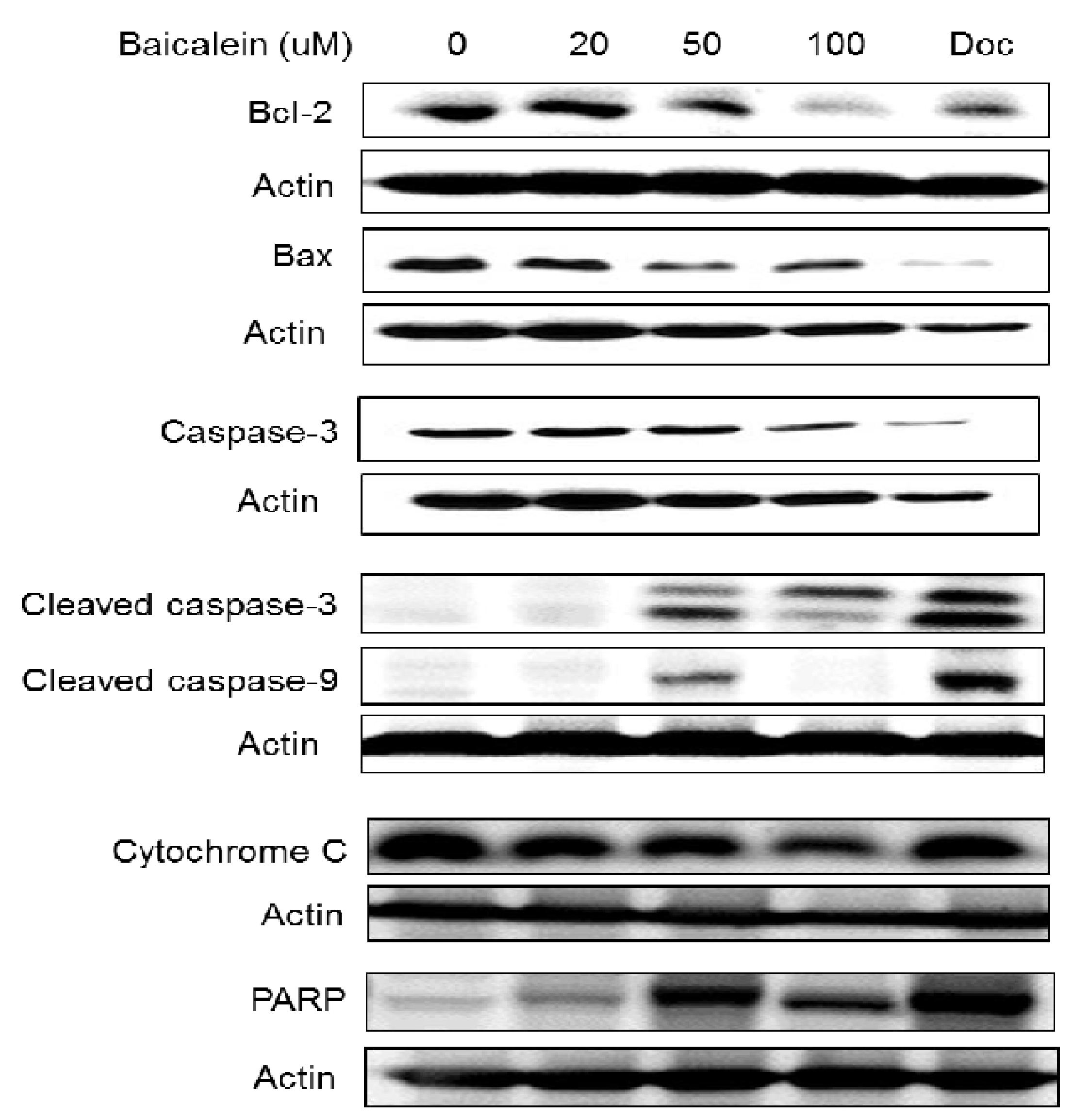

Figure 2. Effect of baicalein on the expression of apoptotic proteins in FRO cells

Cells were treated with baicalein (B) for $48 \mathrm{~h}$. The expression of Bcl-1, Bax, Caspase-3, cleaved caspase-3, -9, cytochrome C, and PARP was determined by Western blot, respectively. Actin was used as an internal control. Doc: docetaxel $50 \mu \mathrm{M}$ as a reference drug.
A)

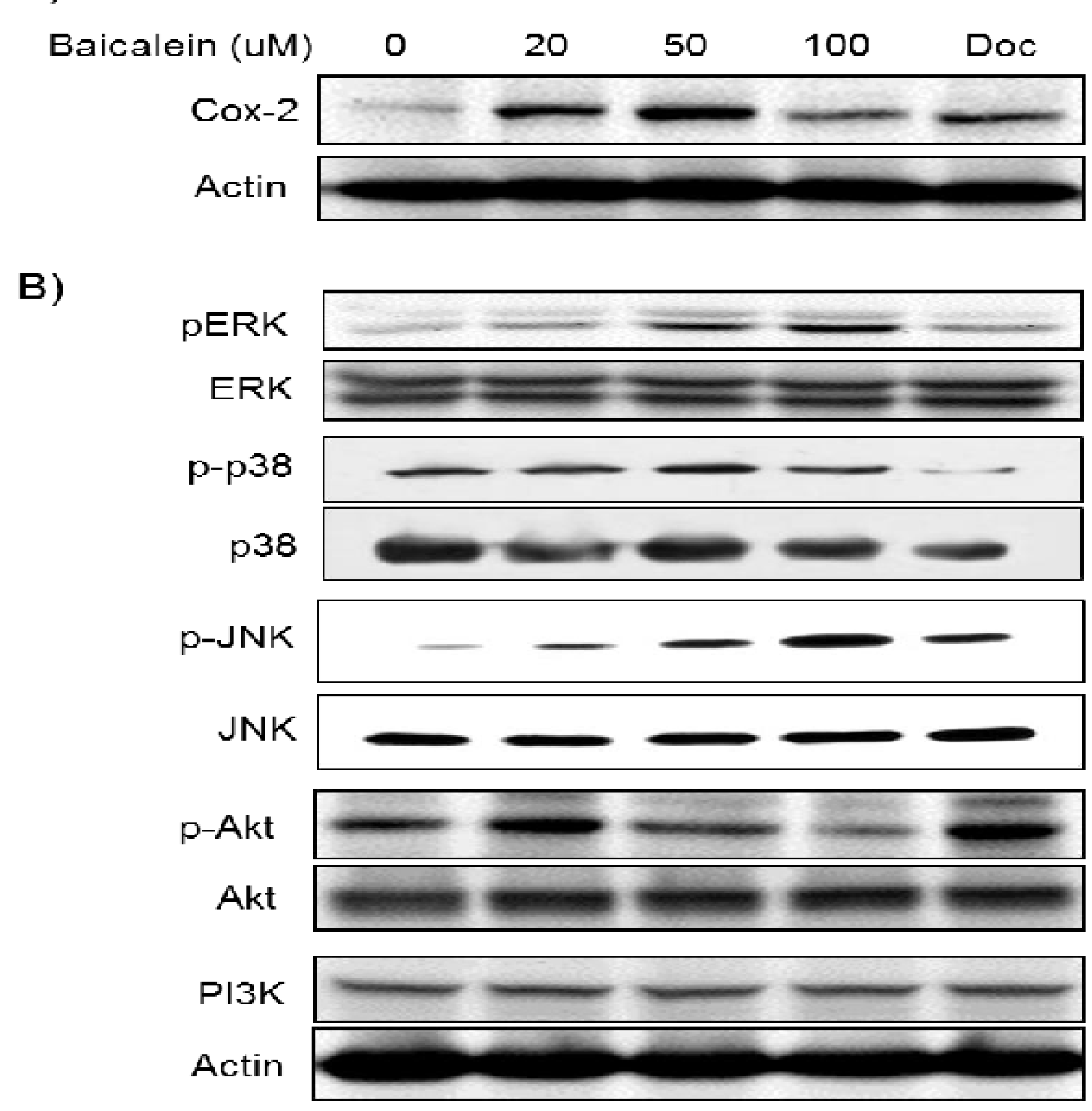

Figure 3. Effect of baicalein on the expression of inflammatory and signal proteins in FRO cells Cells were treated with baicalein (B) for $48 \mathrm{~h}$. The expression of Cox-2 (A), and the phosphorylation of three MAP kineses, ERK, JNK, p38 was determined by Western blot, respectively. Actin was used as an internal control. Doc: docetaxel $50 \mu \mathrm{M}$ as a reference drug.

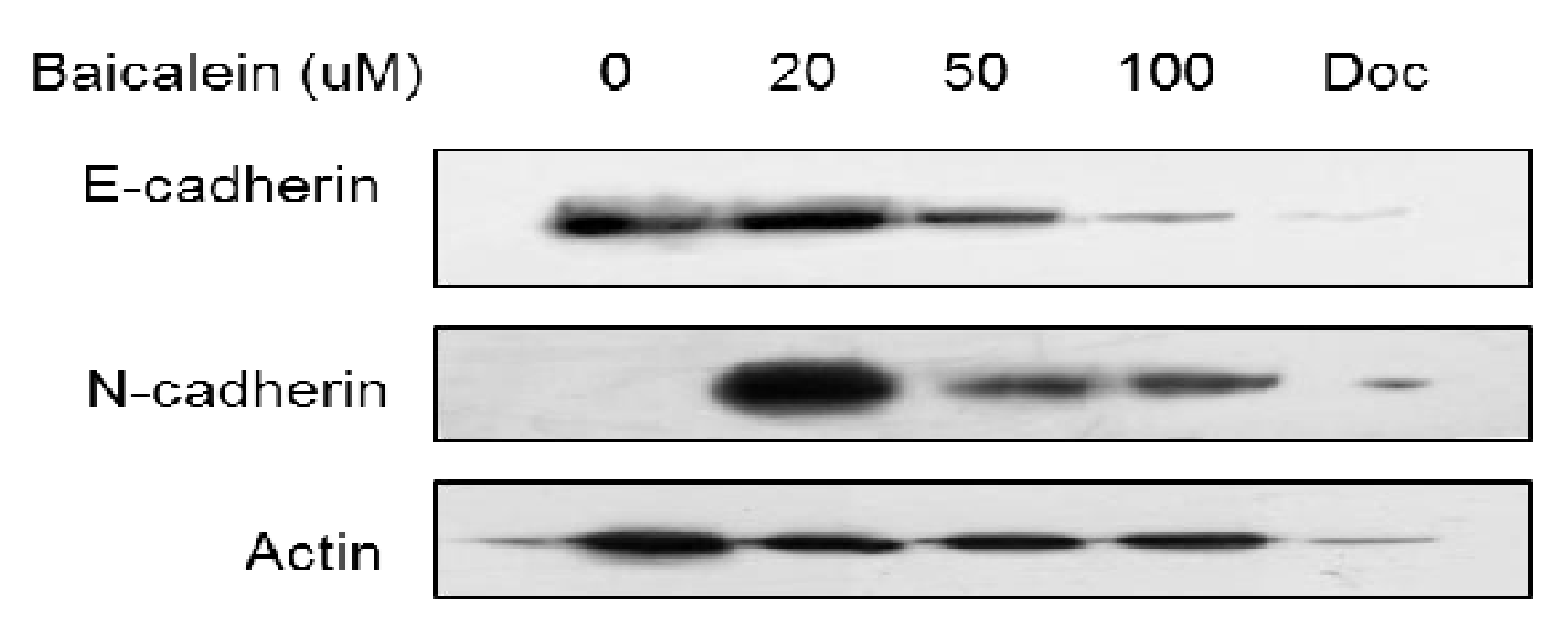

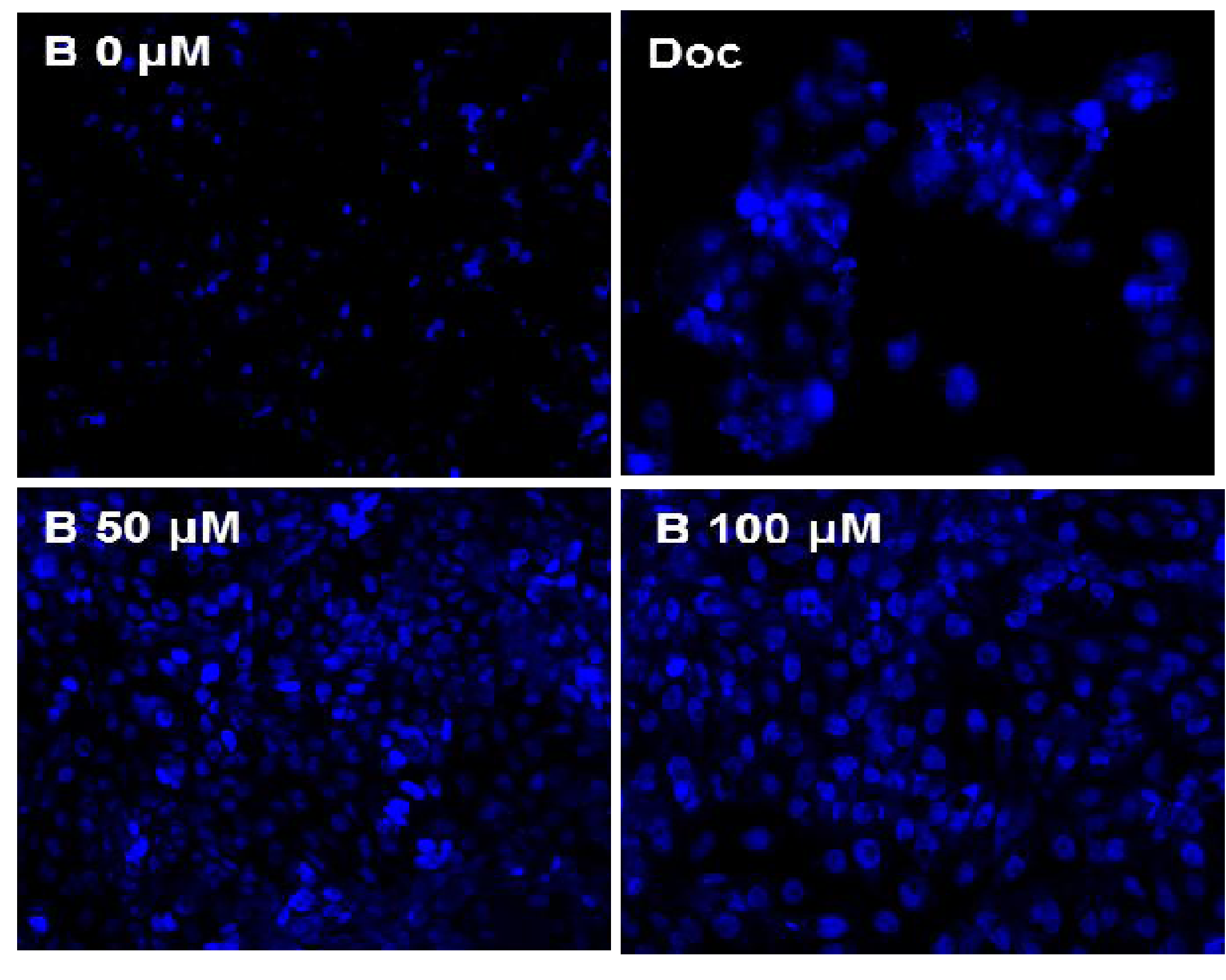

Figure 4. Effect of baicalein on apoptosis in FRO cells

Cells were treated with baicalein (B) for $48 \mathrm{~h}$. The cell apoptosis was determined by Hoechst stain. Doc: docetaxel $50 \mu \mathrm{M}$ as a reference drug.
Figure 5. Effect of baicalein on the expression of Ecadherin and $\mathbf{N}$-cadherin in FRO cells Cells were treated with baicalein (B) for $48 \mathrm{~h}$. The expression of $\mathrm{E}$-cadherin and $\mathrm{N}$-cadherin was determined by Western blot, respectively. Actin was used as an internal control. Doc: docetaxel $50 \mu \mathrm{M}$ as a reference drug.

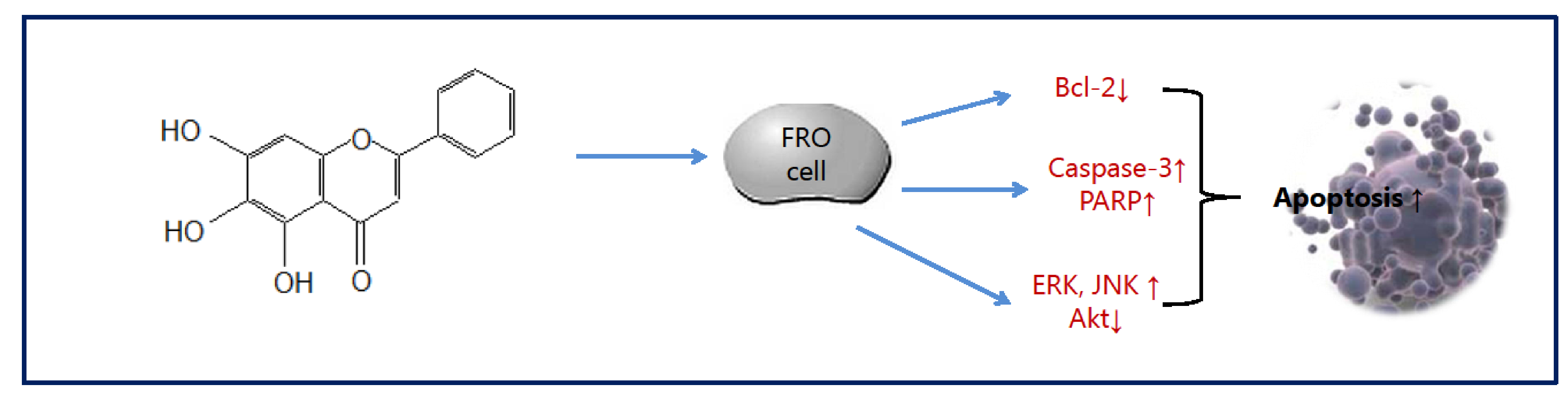

